\title{
HIGH STRENGTH NANOCRYSTALLIZED MULTILAYERED STRUCTURE OBTAINED BY SMAT AND CO-ROLLING
}

\author{
L. Waltz ${ }^{1, a}$, D. Retraint ${ }^{1, b,}$, A. Roos ${ }^{1, c}$, P. Olier ${ }^{2, d}$, J. Lu ${ }^{3, e}$ \\ ${ }^{1}$ Institute Charles Delaunay, FRE CNRS 2848, University of Technology of Troyes, 10000 Troyes, \\ FRANCE \\ ${ }^{2}$ DRT/LITEN/DTNM/LTMEX, Commissariat à l'Energie Atomique de Saclay, 91191 Gif-sur-Yvette, \\ FRANCE \\ ${ }^{3}$ Department of Mechanical Engineering, Hong Kong Polytechnic University, Hung Hom Kowloon, \\ Hong Kong, CHINA
}

alaurent.waltz@utt.fr, ${ }^{b}$ delphine.retraint@utt.fr, ${ }^{c}$ arjen.roos@utt.fr, ${ }^{\text {d} P a t r i c k . O L I E R @ c e a . f r, ~}$ eJian.Lu@inet.polyu.edu.hk

Keywords: Nanocrystalline materials; SMAT; Co-rolling; Multilayered structure, Mechanical properties.

\begin{abstract}
In the present study, a method is presented combining surface nanocrystalline treatment (SMAT) and the co-rolling process. The aim of this duplex treatment is the development of a $316 \mathrm{~L}$ stainless steel semi-massive multilayered bulk structure with improved yield and ultimate tensile strengths, while conserving an acceptable elongation to failure by optimizing the volume fraction and distribution of the nano-grains in the laminate. To characterize this composite structure, tensile tests as well as sharp nanoindentation tests were carried out to follow the local hardness evolution through the cross-section of the laminate. Furthermore, transmission electron microscope (TEM) observations were carried out to determine the correlation between the microstructure, the local hardness and the mechanical response of the structure.
\end{abstract}

\section{Introduction}

Nowadays, ultrafine grain materials with mean grain size smaller than $100 \mathrm{~nm}$ are actively studied and present a widespread interest because they exhibit enhanced mechanical properties, such as high strength, hardness and superplasticity properties. In order to achieve an ultrafine grain structure, several severe plastic deformation (SPD) processes have been proposed, such as accumulative roll-bonding (ARB), equal channel angular pressing (ECAP), high pressure torsion (HPT) or mechanical milling. In addition, numerous studies have shown that recently developed Surface Mechanical Attrition Treatment (SMAT) may induce a grain refinement up to the nanometre scale in the top surface layer of metallic materials, based on mechanisms of severe plastic deformation [1-5]. In the present work, a method is presented combining this SMAT process and the co-rolling process for the development of a semi-massive multilayer bulk structure with improved yield and ultimate tensile strengths, while conserving an acceptable elongation to failure. To characterize this new material, several tests and analyses were carried out on SMATed 316L stainless steel samples and on the resulting hot co-rolled laminate structure. A surface characterization of the treated samples, which was performed simultaneously with local hardness analysis, will be presented. Sharp nanoindentation experiments were performed in order to follow the evolution of the local hardness through the cross-section of specimens and to establish a relation

\footnotetext{
* Corresponding author : Delphine Retraint, delphine.retraint@utt.fr
} 
with grain size distribution. Furthermore, complementary tensile tests highlight the effect of SMAT on the mechanical response of treated and co-rolled samples. The microstructures of SMATed but undeformed tensile specimens have been analysed previously by Transmission Electron Microscopy (TEM) [3,4]. Finally, Scanning Electron Microscopy (SEM) micrographs show how the laminate samples are torn apart during tensile tests.

\section{Experimental procedures}

The material used in this investigation is commercial 316L face-centred cubic (fcc) austenitic stainless steel. For the experimental tests, $120 \times 120 \times 1 \mathrm{~mm}^{3}$ plates with the chemical composition (in wt\%) $0.025 \mathrm{C}, 0.38 \mathrm{Si}, 1.33 \mathrm{Mn}, 0.027 \mathrm{P}, 0.002 \mathrm{~S}, 16.70 \mathrm{Cr}, 2.09 \mathrm{Mo}, 10.20 \mathrm{Ni}, 0.030 \mathrm{~N}, 0.40 \mathrm{Cu}$, $0.07 \mathrm{Co}$ were used. The initial microstructure of the as-received material is characterised by a grain size ranging between 20 and $80 \mu \mathrm{m}$. The surface nanocrystallization of these sheets was obtained by SMAT. The spherical steel shot (material 100Cr6), with a diameter of $3 \mathrm{~mm}$, was placed in a reflecting chamber including an ultrasonic concentrator that was put in motion by an ultrasonic generator. Because of the high vibration frequency of the system $(20 \mathrm{KHz})$, the shot reaches the resonance regime and peens the entire surface of the sample with a high number of impacts in a short period of time. It is noteworthy that impacts during ultrasonic shot-peening are highly random and multidirectional, and the intensity induced by this technique is similar to that of conventional shot-peening processes. In this study, samples have been treated for 30 minutes in air at room temperature. Figure 1 shows the microstructure of a SAMTed sample after electropolishing in a solution of $90 \%$ methanol and $10 \%$ perchloric acid.

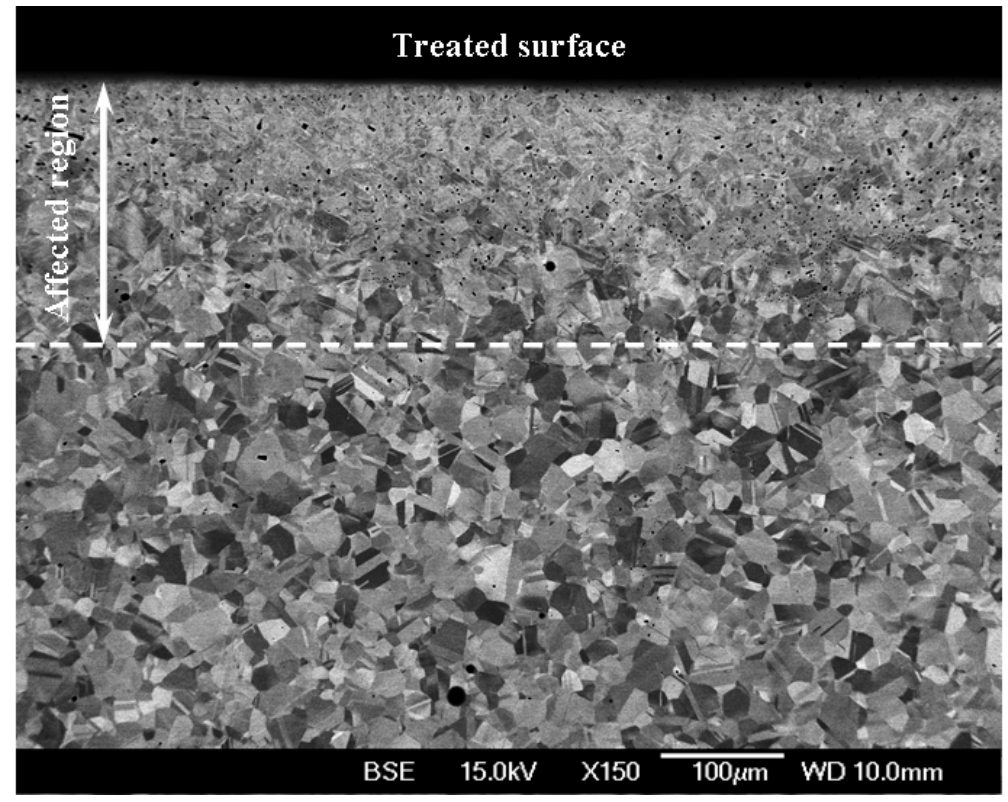

Fig. 1: SEM micrograph of the cross-section of a SMATed 316L stainless steel sample

The co-rolling process at high temperature consists of the hot rolling of three nanocrystallised sheets as illustrated in Fig. 2. The middle plate of the piling has been SMATed on both sides, whereas the two other sheets have been treated on their inner sides only (as depicted in Fig. 2). After preheating treatment at $550^{\circ} \mathrm{C}$ for 60 minutes, the stack of treated sheets was first co-rolled without lubrification. The reduction ratio set by pass was approximately $22 \%$, which corresponds to a global reduction ratio of $66 \%$ after 3 passes. After the first and the second passes, the sample was preheated again for 20 minutes and then after the third pass is water-cooled. The co-rolling was carried out using semi-industrial STANAT reversible quarto rolling equipment under a typical load of about $3500 \mathrm{kN}$. 


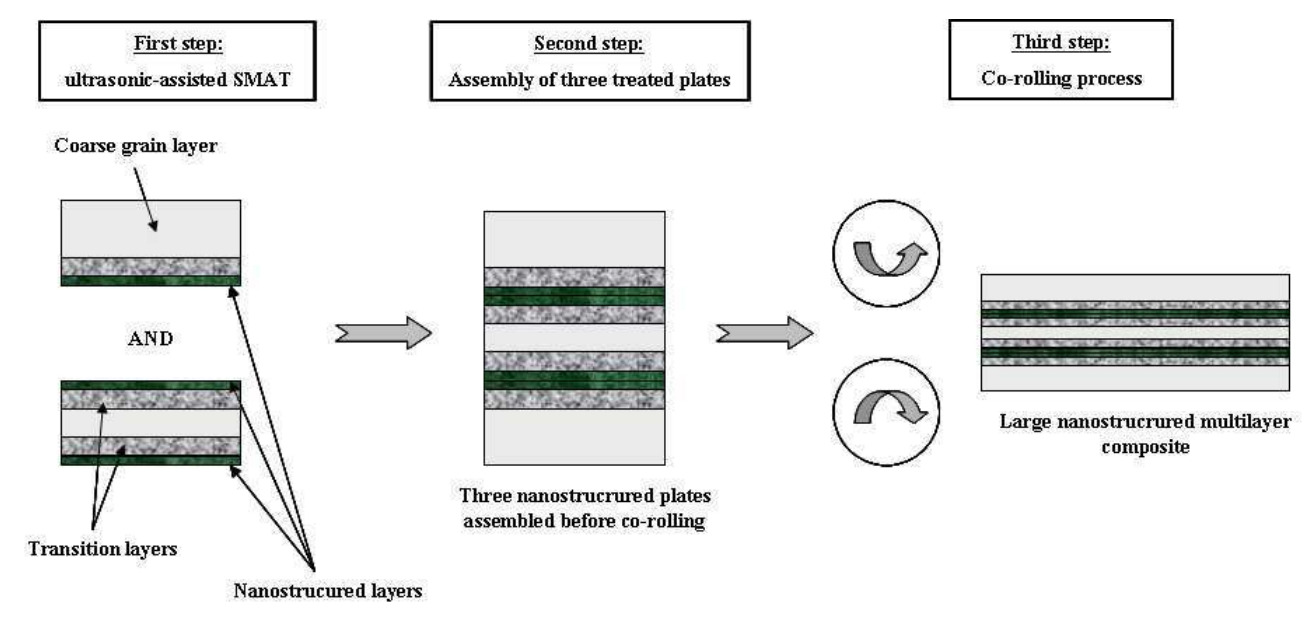

Fig. 2: Global view of the co-rolling process of SMATed samples

The local hardness variation was determined on SMATed samples and on co-rolled laminate using a Nano Indenter $\mathrm{XP}^{\mathrm{TM}}$ fitted with a Berkovich diamond indenter. Furthermore, in order to obtain a precise hardness profile, four indentations were performed at the same distance (depth) from the treated surface. To avoid erroneous hardness measurements due to the affected plastic zone around an indent, the distance between two indentations was fixed to at least $20 \mu \mathrm{m}$. The depth of the indentations themselves was fixed at $600 \mathrm{~nm}$. Before testing, the samples were mechanically polished to mirror finish with $1 \mu \mathrm{m}$ diamond polishing paste.

For a better understanding of the deformation mechanism and the properties of SMATed and corolled stainless steel samples, tensile tests were carried out at room temperature on a screw-driven Kammrath \& Weiss micro-tensile machine at a fixed strain rate of $10^{-4} \mathrm{~s}^{-1}$. The dimensions of the tensile specimens were $36 \mathrm{~mm}$ in total length, a gauge length of $19 \mathrm{~mm}$ and an average cross-section of $1.2 \times 3.6 \mathrm{~mm}^{2}$.

\section{Results and discussion}

In previous studies, microstructural characterizations of SMATed 316L austenitic stainless steel have been carried out [2,3,4,6]. Transmission Electron Microscopy observations (TEM) performed near the extreme top surface of the specimens have thus shown grains in the nanometre range characterized by highly random crystallographic orientations and exhibit an average grain size of approximately $20 \mathrm{~nm}$. SMAT parameters used in this work are similar to the parameters used in the previous studies.

Figure 3 shows the local hardness evolution along the depth of a specimen treated for 30 minutes with $3 \mathrm{~mm}$ shot. It can be observed that the ultrasonic-assisted SMAT induces a considerable enhancement of the local hardness over the cross-section of the sample. The local hardness profile exhibits very high hardness values in the top surface layer of the specimen, values which decrease as the depth from the treated surface increases. For instance, at a depth of 3-5 $\mu \mathrm{m}$, we recorded a very high hardness around $8.5 \mathrm{GPa}$ which was more than 3.3 times higher than the coarse-grain hardness. Moreover, the hardness level reached near the treated top surface in this work was much higher than the hardness proposed by T. Roland et al [4]. The difference can be explained by the position of the indents near the top surface and the depth of each indentation. Indeed, the maximal indentation depth fixed in this study was $600 \mathrm{~nm}$, so that indents could be performed very near the treated surface (between $3 \mu \mathrm{m}$ and $5 \mu \mathrm{m}$ ), while keeping the plastic sphere created under the Berkovich indenter inside the material. Actually, accordingly to the strain and strain rate gradient induced by the SMAT process from the top surface to the strain free matrix, a grain refinement down to the nanometre range is observed in the surface layer. This grain refinement process follows a Hall-Petch-like trend combined with a strain hardening phenomenon of sub-layers and gives a 
simple explanation for the local hardness evolution. In fact, according to this, indents performed in

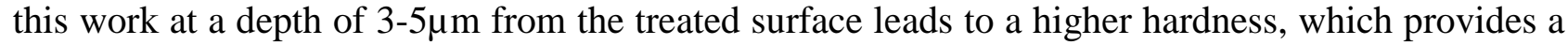
partial explanation for this hardness difference. Considering previous TEM observations, indents performed down to $25 \mu \mathrm{m}$ below the treated surface are supposed to be located in the nanostructured layer (see Fig. 3). However, as pointed out by T. Roland [3], the austenite phase transformation into martensite induced by SMAT has also to be taken into account, so that a part of the high hardness could be attributed to the presence of the hard martensite phase. In addition, it is interesting to note that the hardness recorded in the strain-free matrix of the SMATed sample is slightly higher than the coarse grain hardness, which is certainly a hardening effect induced by the distortion of the sample during SMAT.

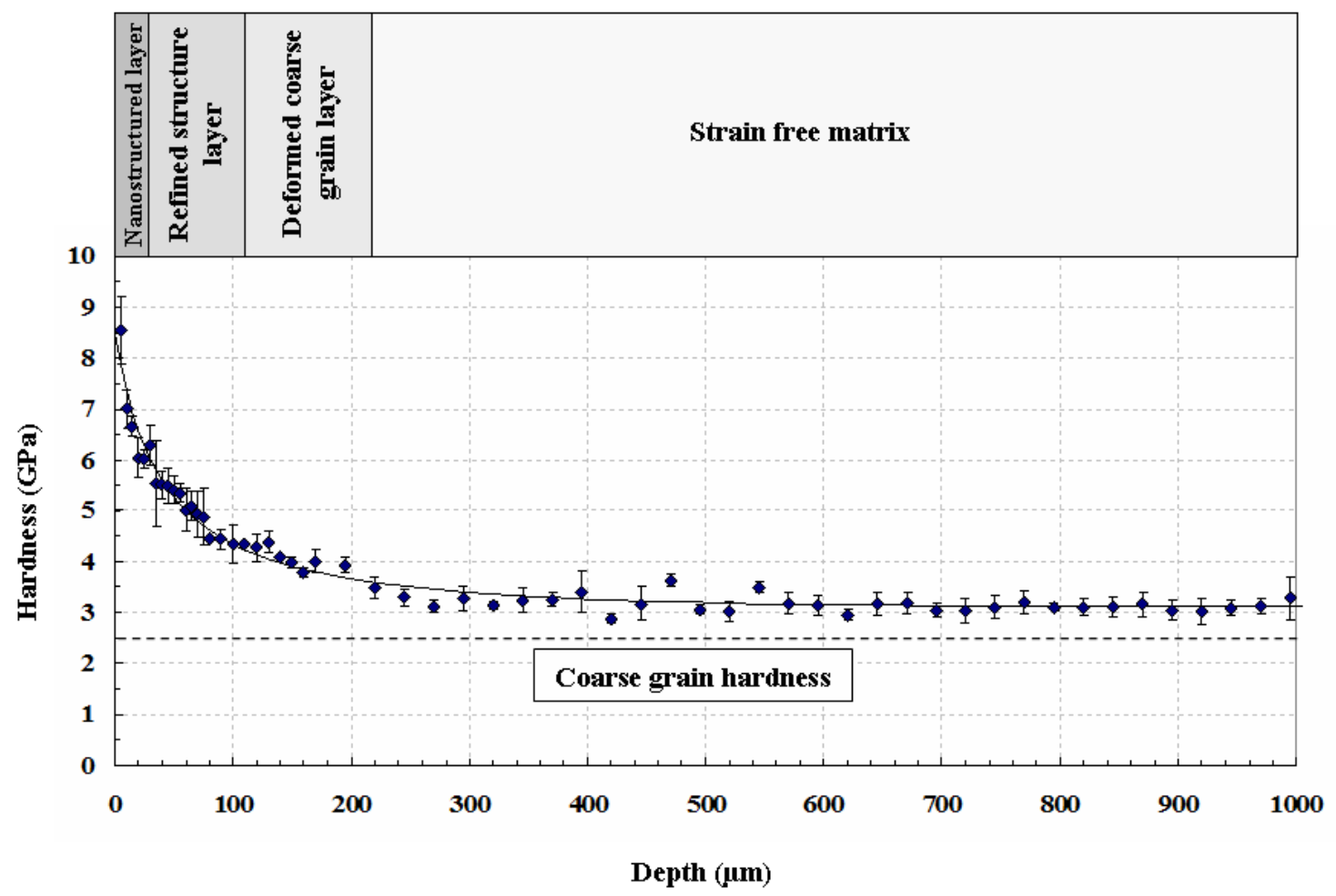

Fig. 3: Local hardness measurements obtained by nanoindentation tests on a SMATed sample treated for 30 minutes with $3 \mathrm{~mm}$ shot on one side

Fig. 4 shows engineering stress-strain curves of a co-rolled multilayer laminate, an as-received specimen and a SMATed sample (30 minute treatment with $3 \mathrm{~mm}$ shot). It clearly shows that the SMAT process considerably affects the yield stress of the treated materials, whereas the ductility does not seem to be significantly changed, since an elongation to failure of $36 \%$ is reached. This sample exhibits a yield strength value as high as $525 \mathrm{MPa}$, which corresponds to an increase of the yield strength of about $78 \%$ compared to the coarse grain sample (295MPa). This strength increase can be directly explained by the presence of SMAT-induced nanostructured layers on the top surface of both sides of the specimen, which are able to sustain a large part of the load. Furthermore, by analysing this figure, it clearly appears that SMAT combined with the co-rolling process leads to a high-strength co-rolled multilayer structure. Indeed, despite a reduction of the elongation to failure, the co-rolled laminate presents high yield and ultimate strengths reaching respectively $800 \mathrm{MPa}$ and $914 \mathrm{MPa}$. We cannot claim, that compared to the SMATed sample, the corolled specimen provides a very high strength level, since we recorded a yield strength increase of $53 \%$. It is noteworthy that the rolling process leads to the reduction of the ductility of the structure through work hardening of the material, which reaches an elongation to failure of $13 \%$. Although this ductility reduction is induced by the combined SMAT-rolling process, the elongation to failure of the co-rolled specimen stays acceptable. The high strength of the multilayer structure can be 
explained by the combination of an increased volume fraction of nano and ultrafine grains, which are able to sustain a great part of the load, combined with a hardening effect induced by co-rolling.

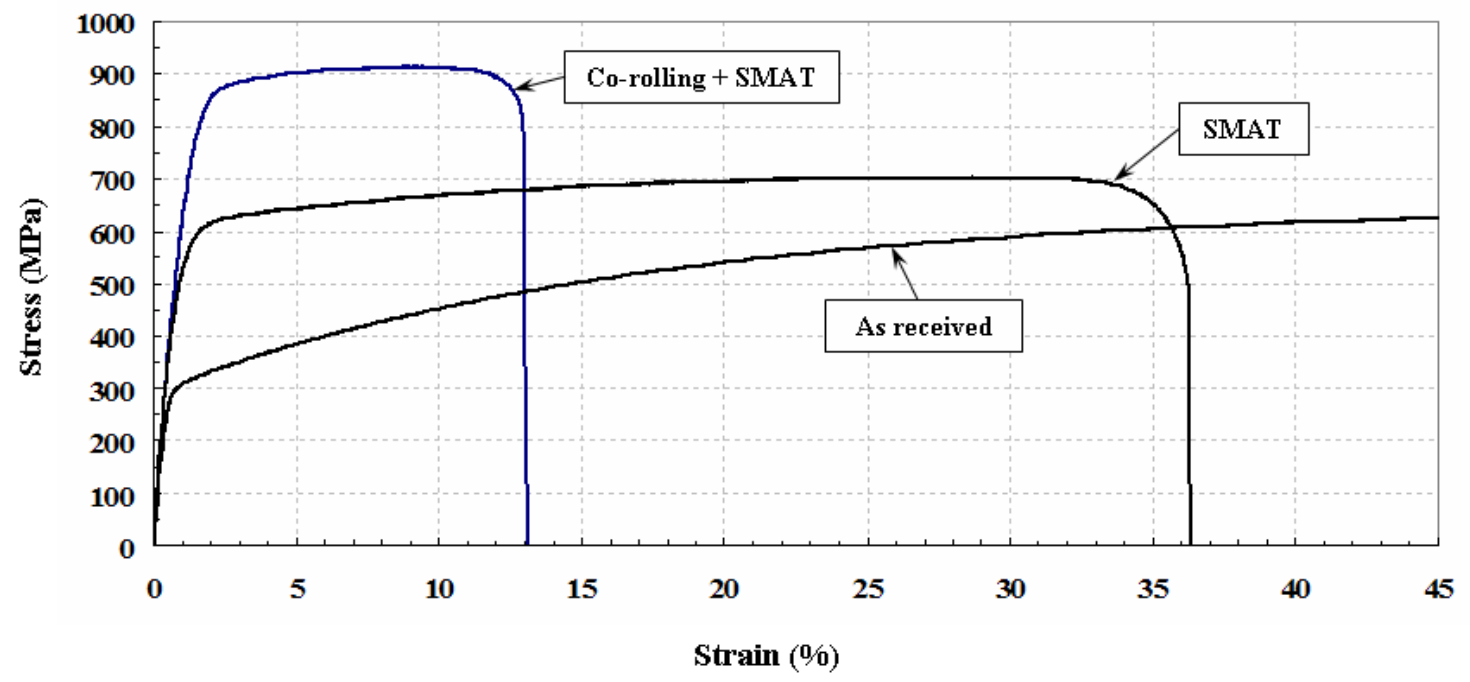

Fig. 4: Uniaxial engineering tensile stress-strain curves of (1) as-received coarse grain sample, (2) SMATed specimen, (3) SMAT + co-rolled specimen

The SEM fractographs in Fig. 5 show an example of the fracture surface of the co-rolled multilayer tensile specimen after the tensile test. The fracture morphology of this sample presents a significant feature, since on the two lateral sheets, initially SMATed on their inner sides, two distinct failure structures can be clearly distinguished. Indeed, Fig. 5 b) presents a high magnification SEM micrograph of such an area showing a clear and straight separation between the two regions, both characterized by a specific failure mode. It is interesting to note that the flat fracture surface region near the interface (region A in Fig. 5) corresponds well with the initially nanostructured layers after SMAT $[2,3,4]$. Furthermore, this flat failure surface is reminiscent of a glassy brittle material, whereas the outer side of the first sheet and the inner side of the middle sheet present a heavily dimpled morphology (region B in Fig. 5 b)), characteristic of a ductile material. These observations clearly indicate that this sample was torn apart in a non-trivial combined ductile-brittle manner. In addition, it is interesting to note that in the last stage of deformation during the tensile tests, local debonding occurs in the striction zone (Fig. 5 a).
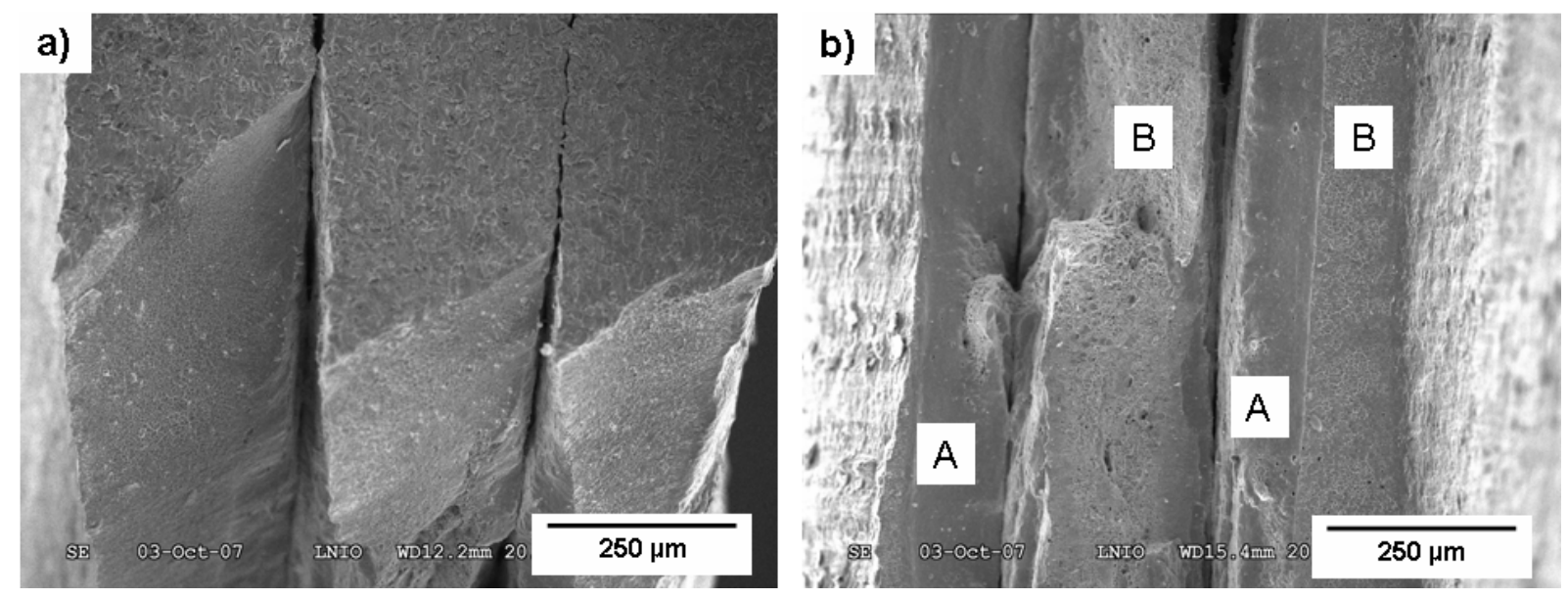

Fig. 5: a) SEM micrographs of the fracture surface of the co-rolled multilayer tensile specimen; $b$ ) high magnification SEM micrographs of selected regions (white square) 


\section{Conclusion}

In the present study, generation of a high strength semi-massive multilayer structure by a combination of Surface Mechanical Attrition Treatment (SMAT) and the co-rolling process at high temperature was investigated. To characterize SMATed and co-rolled laminate, local hardness measurements, tensile tests and electron microscopy observations were performed. It has been shown that the SMAT process has a significant influence on local hardness evolution through the cross-section of the sample and values recorded near the treated top surface are very high $(\approx 8,5$ $\mathrm{GPa})$. The hardness increase near the surface can be attributed to the presence of an SMAT-induced nanocrystalline layer, which is confirmed by an enhanced yield strength obtained by tensile tests. Furthermore, the new co-rolled structure presents improved yield and ultimate strengths $(800 \mathrm{MPa}$ and $914 \mathrm{MPa}$ respectively), while keeping an acceptable strain to failure of approximately $13 \%$. Scanning electron micrographs of the failure surface obtained after tensile tests clearly show a separation between flat and dimpled areas, indicating that the specimen was torn apart in a ductilebrittle manner.

\section{References}

[1] K. Lu, J. Lu, Surface nanocrystallization (SNC) of materials and its Effects on Mechanical Behavior, Encyclopedia of Comprehensive Structural Integrity (CSI), 2002, Vol.(8), p. 1-35.

[2] T. Roland, D. Retraint, J. Lu, K. Lu, Generation of nanostructures on 316L stainless and its effect on mechanical behaviour, 2005, Mater. Sci. Forum, Vol. (490-491), p. 625-630.

[3] T. Roland, D. Retraint, J. Lu, K. Lu, Fatigue life improvement through nanostructuring of stainless steel by means of surface mechanical attrition treatment, 2006, Scripta Mater., 54, 1949-1954.

[4] T. Roland, D. Retraint, J. Lu, K. Lu, Enhanced mechanical behavior of a nanocristallised stainless steel and its thermal stability, 2007, Mater. Sci. Eng., A445-446, p. 281-288.

[5] N.R. Tao, M.L. Sui, J. Lu, and K. Lu, Surface nanocrystallization of iron induced by ultrasonic shot peening, NanoStruc. Mater., 1999, Vol. (11), p. 443-440.

[6] G. Liu, J. Lu, and K. Lu, Surface nanocrystallization of 316L stainless steel induced by ultrasonic shot peening, Mater. Sci. Eng., 2000, A 286, p. 91-95.

[7] X.H. Chen, J. Lu, L. Lu, K. Lu, Tensile properties of a nanocrystalline 316 austenitic stainless steel, Scripta Mater., 2005, Vol. (52), p. 1039-1044. 